\title{
Drei molekulare Marker für fünf Hauptgruppen
}

Fragestellung: Wie viele molekulare Marker braucht man für die Klassifikation von Gliomen?

Hintergrund: Die WHO-Klassifikation der primären Hirntumoren basiert aktuell ausschließlich auf morphologischen Kriterien. In den letzten Jahren ist jedoch deutlich geworden, dass sich Tumoren ähnlicher Histologie oft sehr deutlich bezüglich der Prognose und des Ansprechens auf Therapie unterscheiden. Dies ist insbesondere bei der Untersuchung von Patienten mit Oligodendrogliomen in klinischen Studien deutlich geworden. Die vorliegende Arbeit basierte auf der Hypothese, dass sich mittels der Bestimmung des TERT-(Telomerase, reverse Transkriptase)-

Promoter-Mutationsstatus,

Eckel-Passow JE, Lachance DH, Molinaro AM et al. Glioma Groups Based on 1p/19q, IDH, and TERT Promoter Mutations in Tumors. N Engl J Med 2015; 372: 2499-508 des Isozitratdehydrogenase (IDH)-1/2-Mutationsstatus und des $1 \mathrm{p} / 19 \mathrm{q}$-Kodeletionsstatus fünf Gruppen von Gliomen definieren lassen, die klinisch und molekular sehr homogen sind.
Patienten und Methodik: Die Autoren untersuchen 1.087 Gliome mit Information zu diesen Markern. Zudem wurden die Gliompatienten mit 11.590 Kontrollen bezüglich von mit Gliomerkrankung assoziierten Keimbahnvarianten korreliert.

Ergebnisse: Unter den 615 Patienten mit WHO-Grad-II/III-Gliomen hatten $29 \%$ alle drei Veränderungen, $5 \%$ hatten TERTund IDH-Mutationen, $45 \%$ hatten nur IDH-Mutationen, $7 \%$ hatten keine der drei Mutationen, und $10 \%$ hatten nur TERTMutationen, 5\% hatten andere Kombinationen. Bei den 472 Glioblastomen waren weniger als $1 \%$ triple-positiv, $2 \%$ hatten TERT- und IDH-Mutationen, $7 \%$ hatten nur IDH-Mutationen, $17 \%$ waren triple-negativ, und $74 \%$ hatten nur TERT-Mutationen. Die molekularen Gruppen waren mit unterschiedlichem Überleben und spezifischen Keimbahnvarianten assoziiert.

Schlussfolgerungen: Gliome lassen sich in fünf Hauptgruppen einteilen, wenn man die drei genannten Marker bestimmt. Die Gruppen unterscheiden sich in Alter, Überleben und Assoziation mit Keimbahnvarianten.

\section{Neue Studien mit homogeneren Patientenpopulationen durchführen}

Aufgrund der Limitationen der rein morphologisch definierten WHO-Klassifikation gibt es zahlreiche Ansätze der molekular basierten Klassifikation von Gliomen. Die vorliegende Arbeit stützt sich auf drei molekulare Veränderungen, 1p/19q, IDH und TERT-Promoter, die als etabliert gelten. Die Einteilung in fünf Gruppen ist allerdings sehr komplex und wird mutmaßlich wenig Einfluss auf die anstehende Revision der WHO-Klassifikation haben, weil ein sehr viel einfacherer und bereits weitgehend etablierter Algorithmus mit zwei molekularen Biomarkern auskommt, nämlich IDH- und 1p/19q-Status [1, 2]. Die aktuelle Arbeit bleibt den Nachweis schuldig, dass die komplexe Gliederung, die hier vorgeschlagen wird, gegenüber der etwas praktikabler wirkenden Einteilung der Gliome in drei Gruppen Vorteile aufweist. Letztlich werden aber prospektive klinische Studien notwendig sein, um den Wert der neuen Gliomklassifizierungen zu prüfen, denn auch die bisherigen Ansätze der Reklassifikation waren im Wesentlichen

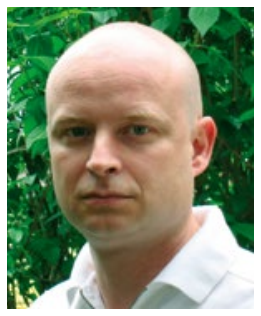

Prof. Dr. med. Michael Weller,

Zürich/Schweiz

Direktor der Klinik für Neurologie,

Universitätsspital Zürich

E-Mail: michael.weller@usz.ch durch klinische Studienergebnisse getrieben, nämlich die Beobachtung, dass Patienten mit Tumoren mit IDH-Mutation, und unter diesen insbesondere die mit 1p/19q-Kodeletion, eine deutlich bessere Prognose aufweisen. Ungeachtet dessen trägt die vorliegende Arbeit aber dazu bei, den Stellenwert molekularer Diagnostik bei Gliomen herauszuarbeiten und die Forderung zu untermauern, dass zukünftige klinische Studien mit homogeneren Patientenpopulationen durchgeführt werden sollten.

\section{Referenzen:}

1. Weller M et al. Acta Neuropathol 2015; 129: 679-93

2. The Cancer Genome Atlas Research Network. N Engl J Med 2015; 372: $2481-98$

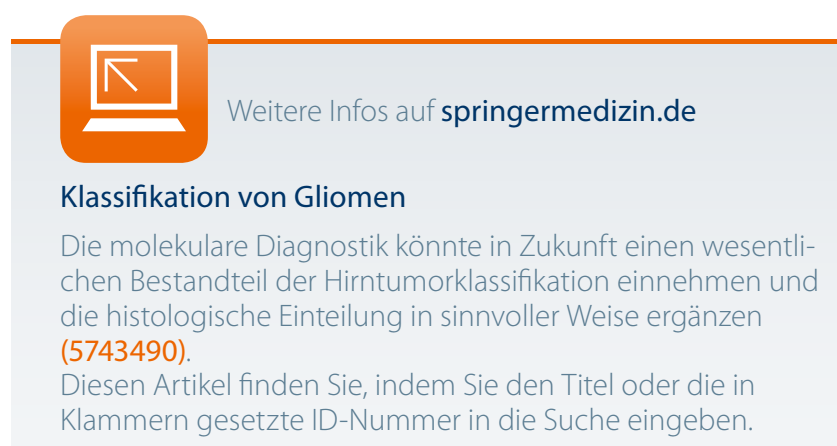

